\title{
Development of mathematical model of laser treatment heat processes using diffractive optical elements
}

\author{
S.P. Murzin ${ }^{1}$, A.Yu. Tisarev ${ }^{1}$, M.V. Blokhin ${ }^{1}$, S.A. Afanasiev ${ }^{1}$ \\ ${ }^{1}$ Samara National Research University, 34 Moskovskoe Shosse, 443086, Samara, Russia
}

\begin{abstract}
Calculation of laser beam intensity distribution in the focal plane of diffractive optical element was performed using software TracePro. To determine temperature fields occurring in the process of laser treatment of material, software of computational gas dynamics CFX version 15.0 and supercomputer "Sergey Korolev" were used. Temperature dependence of heat conductivity for the coating of the Ni-Al alloy produced by plasma spraying was determined. Alloy heat capacity was calculated based on additive rule. Besides, the temperature dependence of the absorption coefficient at $\mathrm{CO}_{2}$-laser treatment was also determined.
\end{abstract}

Keywords: diffractive optical elements; laser treatment; mathematical model; heat source; temperature

\section{Introduction}

Coating deposition on the components of gas turbine engine requires a sublayer. The sublayer is an intermediate tie coat, which compensates for differences in the coefficient of linear expansion of materials, as well as provides a higher adhesion strength. As reacting or thermoreacting nickel-aluminum powder is used as such material between the substrate and the sprayed coating. Thermally reactive powder is sprayed on the substrate by the method of plasma spraying. In this method, powder particles interact with a high-temperature plasma jet [1-3]. When the exothermically reacting powder is sprayed, its components react with the formation of new compounds and a significant amount of heat is released, that allows additionally heat the powder. To intensify the diffusion of the composite components of material, at least one component must remain in the liquid or gaseous phase.

To improve coatings characteristics, the heat treatment of sprayed coating is widely used [4-6]. Heat treatment is applied to improve the coating characteristics by following factors: an increase in the contact area of the coating and the substrate; reduction in material porosity; increase in the strength of interparticle bonds [7, 8]. Laser treatment of sprayed coatings is one of the methods to improve coating properties [9-12]. Heat treatment of the sprayed coating can cause material cracking due to an increase the level of stresses during phase transformations. However, it is possible to realize laser treatment modes leading to a decrease in residual stresses. On purpose to reduce residual stresses in the absence of crack formation, the speed of laser spot moving along the substrate surface and the beam power density must be determined.

For the formation of the laser beam, various optical systems are used. However, none of them can provide the appropriate combination of such properties as the creation of the required power distribution, the concentration of the all energy of laser beam in the treatment zone of a given shape, and high reliability. The use of diffractive optical elements is promising [13-17]. Diffractive optical elements make it possible to form a predetermined beam intensity profile in the focal plane, carrying out the transformation of laser energy, chosen by calculation. The use of diffractive optical elements in the technology of laser material treatment reveals new possibilities for controlling the properties and operational characteristics of processed parts [18-21].

The aim of this work is to develop the mathematical model of laser treatment processes of the sublayer at coating deposition on the corps parts of a gas turbine engine using diffractive optical elements. It is known, temperature cycle can be a factor, which largely determines the state of processes in the treated materials. Similar approaches for mathematical model design can be used to study the formation of nanostructured materials by laser treatment.

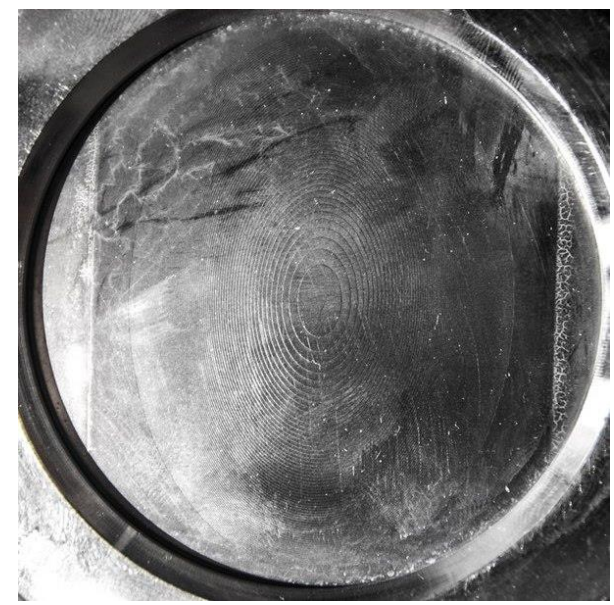

Fig. 1. Appearance the working surface of DOE. 


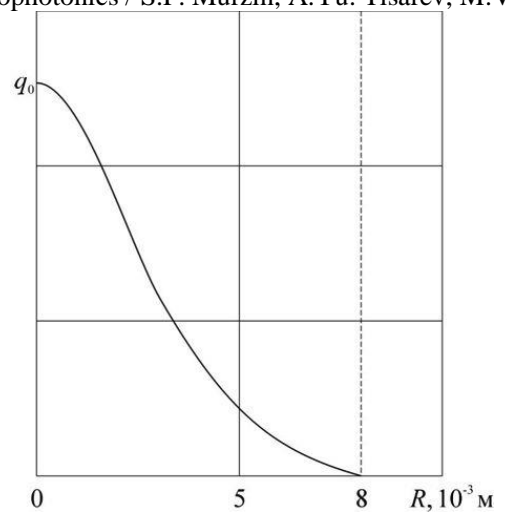

Fig. 2. Beam power density distribution of a CO2 slab laser Rofin DC 010.

\section{Calculation of the laser beam intensity distribution in the DOE focal plane}

The laser beam intensity distribution in the DOE focal plane has been calculated. It has the following parameters: $f=0.2241$ $\mathrm{m} ; L_{0}=5.6 \cdot 10^{-3} \mathrm{~m} ; R=2.5 \cdot 10^{-2} \mathrm{~m} ; r=0.7 R$. The DOE working surface is shown in Fig. 1 . The diameter of the focused beam of the Rofin DC $010 \mathrm{CO}_{2}$ slab laser is $1.6 \cdot 10^{-2} \mathrm{~m}$. The beam power is regulated within $10 \ldots 1000 \mathrm{~W}$. Beam wavelength is $\lambda=$ $10.6 \cdot 10^{-6} \mathrm{~m}$. The beam power density distribution is shown in Fig 2. This distribution is close to Gaussian distribution: the beam quality parameter or beam distribution parameter is $M^{2}=1.1$. To change the size of laser beam, focused by DOE, it is possible to use a telescopic system of two lenses.

For calculations the software TracePro was applied. This software is designed for three-dimensional modeling of optical components surfaces, construction of the path of beams in optical systems and optical analysis. TracePro software make it possible to design the optical elements according to the equations of their surfaces. In TracePro realized the method of generalized ray tracing. Calculation of each beam incident on the surface of the optical element is performed taking into account absorption, reflection, refraction, diffraction and scattering.

The surface of the diffractive optical element is designed using the macro-language built in the TracePro software. It refers to the type of schematic programming languages that allow to compose macroprograms using loop and branch operators. To design an optical surface at $f=0.2241 \mathrm{~m} ; L_{0}=5.6 \cdot 10^{-3} \mathrm{~m}$, the value of the polynomial was determined, in the form of which it is possible to represent the relation:

$$
P n=\frac{\int_{0}^{M \cos \theta} d U \int_{0}^{\sqrt{R^{2}-U^{2}}} \exp \left(-\frac{U^{2}+V^{2}}{r^{2}}\right) d V}{\pi f(\vartheta(R / r))^{*}}
$$

In Matlab software it was determined:

$P n=-2.3267 \cdot 10^{-18} \cdot x^{6}+5.7104 \cdot 10^{-15} \cdot x^{5}-5.8706 \cdot 10^{-12} \cdot x^{4}+2.4413 \cdot 10^{-9} \cdot x^{3}+1.0053 \cdot 10^{-6} \cdot x^{2}+4.4895 \cdot 10^{-5} \cdot x-0.00086771$.

The calculated density distribution along the axis $O y$ in the DOE focal plane at a beam power of $Q=500 \mathrm{~W}$ is shown in Fig.3. We represent $q(x, y)$ in the form of equation

$$
\begin{aligned}
q(\xi, \eta) & =q_{0}\left(a_{n_{1}} \bar{\xi}^{2 n}+a_{n_{1}-1} \bar{\xi}^{2(n-1)}+\ldots+a_{2} \bar{\xi}^{4}+a_{1} \bar{\xi}^{2}+a_{0}\right) \cdot \\
& \cdot\left(b_{n_{2}} \bar{\eta}^{2 m}+b_{n_{2}-1} \bar{\eta}^{2(m-1)}+\ldots+b_{2} \bar{\eta}^{4}+b_{1} \bar{\eta}^{2}+b_{0}\right)
\end{aligned}
$$

where $q_{0}$ is the power density in the center of the heat source; $\bar{\xi}=\xi /\left(10^{-3} \mathrm{~m}\right) ; \bar{\eta}=\eta /\left(10^{-3} \mathrm{~m}\right)-\operatorname{dimensionless}$ coordinates; ; $a_{n_{1}}, a_{n_{1}-1}, \ldots, a_{2}, a_{1}, a_{0} ; b_{n_{1}}, b_{n_{1}-1}, \ldots, b_{2}, b_{1}, b_{0}$ - coefficients of polynomials, where $n$ and $m$ are integers; $v(\xi, \eta)-$ is an additional function.

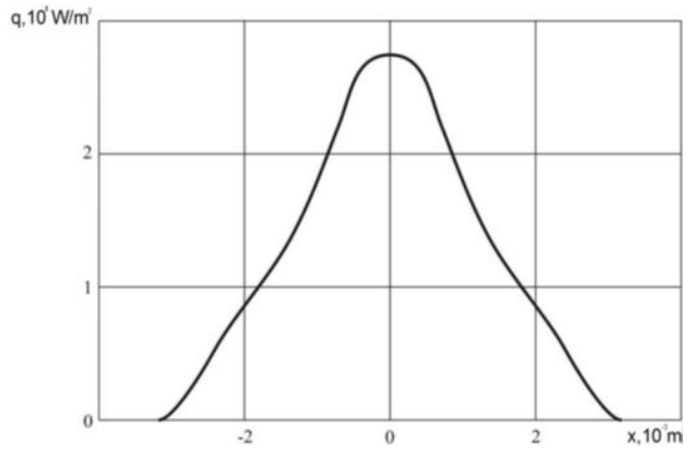

Fig. 3. Intensity distribution along the axis $O y$ in the DOE focal plane at beam power $Q=500 \mathrm{~W} ; q_{0}=2,6504 \cdot 10^{8} \mathrm{~W} / \mathrm{m}^{2}$. 
We take $a=6.25 \cdot 10^{-3} \mathrm{M} ; b=1.25 \cdot 10^{-3} \mathrm{M} ; n_{1}=5 ; n_{2}=2 ; a_{5}=-4.7423 \cdot 10^{-5} ; a_{4}=1.6906 \cdot 10^{-3} ; a_{3}=-2.2028 \cdot 10^{-2} ;$ $a_{2}=0.13326 ; a_{1}=-0.44559 ; a_{0}=1 ; b_{2}=15.13 ; b_{1}=-7.2412 ; b_{0}=1 ; q_{0}=2.6504 \cdot 10^{8} \mathrm{~W} / \mathrm{m}^{2}, v(\xi, \eta)=1.0 . \xi=x ; \eta=y$.

We have obtained an expression describing the power density distribution $q(x, y)$ in the form of equation for a strip heat source at beam power of $Q=500 \mathrm{~W}$ :

$q(x, y)=q_{0}\left(-4.7423 \cdot 10^{-5} \cdot x^{10}+1.6906 \cdot 10^{-3} \cdot x^{8}-2.2028 \cdot 10^{-2} \cdot x^{6}+0.13326 \cdot x^{4}-0.44559 \cdot x^{2}+1\right)$. $\cdot\left(15.13 \cdot y^{4}-7.2412 \cdot y^{2}+1\right)\left[\mathrm{W} / \mathrm{m}^{2}\right]$

at $q_{0}=2.6504 \cdot 10^{8} \mathrm{~W} / \mathrm{m}^{2} ; x \in[-3.125 \mathrm{~mm} ; 3.125 \mathrm{~mm}] ; y \in[-0.625 \mathrm{~mm} ; 0.625 \mathrm{~mm}]$.

An experimental determination of the laser beam power density distribution using DOE has been performed. To measure the power density distribution in the spot of the Rofin DC $010 \mathrm{CO}_{2}$ slab laser, a mechanical scanning method was applied. A standard power meter equipped with a square diaphragm with size of $10^{-4} \times 10^{-4} \mathrm{~m}$ was used for these purposes. Results obtained in experimental researches correlate good with the calculated data. The relative error in determining the power density $q$ did not exceed $5 . . .7 \%$.

\section{Construction of a mathematical model of the heat processes of laser treatment of the sublayer during at spraying of a triggered coating on the body parts of a gas turbine engine using DOE}

To determine the temperature fields on the supercomputer "Sergey Korolev" both in the substrate and coating occurred at the laser treatment of material, software CFX 15.0 was used. To solve the problem, a finite-element model of the all coated working ring was built. To simulate cooling due to radiation and convective heat transfer, an air domain model was built. The calculation scheme is shown in Fig. 4.

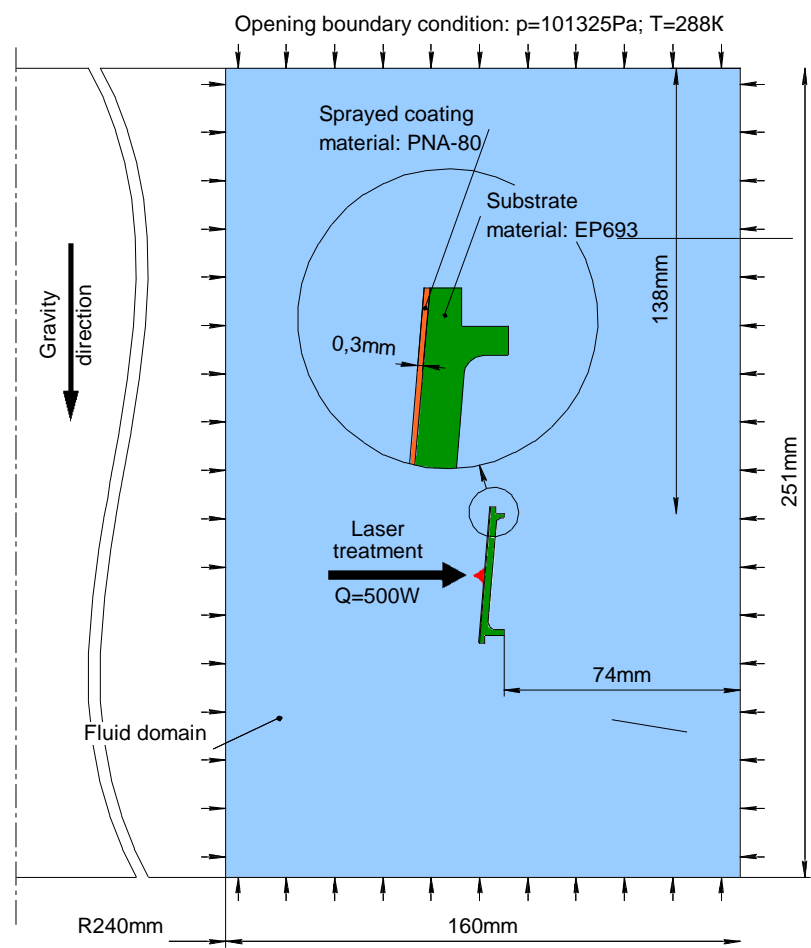

Fig. 4. Calculation scheme for determining the temperature fields in the substrate and coating.

Models of the coating and substrate for the sector at $30^{\circ}$ were subdivided into hexagonal elements with an element edge size $2.5 \cdot 10^{-4} \mathrm{~m}$ (Fig. 5a), while the remaining volume of rings consisted of tetrahedral elements. Air domain was subdivided by a tetrahedral grid as well. The region of the near-wall layer of air domain contained hexagonal elements, represented in Fig. 5b. Directly in the laser treatment area, the size of the elements of finite-volume was reduced.

Turbulence model SST was applied for these calculations. In this case the flow is characterized by low Reynolds numbers, and therefore for the correct simulation of detached flows, the values of the parameter $y+$ are less than 1 . These values $y+$ were achieved by choosing the geometrical characteristics of the finite-volume elements in the near-wall layer. The radiation from the walls was taken into account by connecting the Discrete Transfer model. Radiation characteristics of the surface were described by a gray body model. The upward movement of heated air due to a reduction in its density was taken into account by the Buoyant model.

The model had the following boundary conditions. The heat flow power was $500 \mathrm{~W}$. Heat flow of the laser source was determined by 125 heat source points with increment $2.5 \cdot 10^{-4} \mathrm{~m}$. To simulate the convective cooling of the technological object, the opening air boundary was simulated in Fig. 4. In the calculation model, it was possible to change the rotational speed of the treated body. In order to determine the temperature distribution of the ring during laser treatment, the following physical properties of the coating and substrate materials have to be specified: density, heat conductivity and heat capacity. In addition, to simulate the absorption of heat flow and ring cooling, it is necessary to determine the beam characteristics of the surface. 

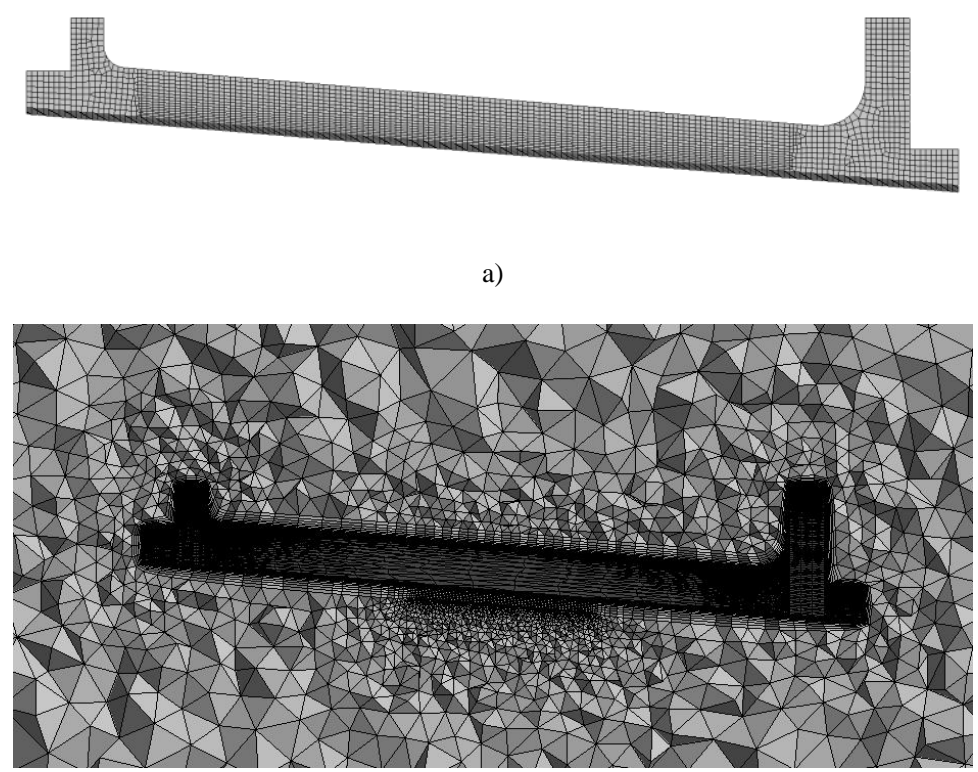

b)

Fig. 5. Discretization of the calculated area: model of the coating and substrate (a); model of air domain (b)

At coating deposition the interaction of its constituent components is accompanied by chemical reactions that lead to the formation of material physical properties, which are different from original components. The change in the properties of materials as a function of temperature is not given with sufficient accuracy in known monographs and reference books. It led to the need for their calculation. The dependence of the heat conductivity on the temperature for coatings made of Ni-Al alloy obtained by plasma spraying in air was determined. Calculation of heat capacity $\mathrm{Ni}-\mathrm{Al}$ alloy was performed based on the additivity rule. The temperature dependence of absorption coefficient under the action of $\mathrm{CO}_{2}$-laser was determined. It was necessary for estimating the amount of absorbed energy.

\section{Conclusion}

The calculation of intensity distribution of laser beam in the focal plane of the DOE was conducted. The software complex TracePro is used for calculation, which allows to build optical elements by the equations of their surfaces. The density distribution in the DOE focal plane at beam power of $500 \mathrm{~W}$ was determined. An expression is obtained that describes the power density distribution in the form equation for a strip heat source. The experimental determination of the laser beam power density distribution using DOE has been performed. A CO2 slab laser Rofin DC 010 was used. His the beam propagation parameter is 1.1. It is determined that the researchers of experimental studies correlate well with the calculated data. The relative error in determining the power density did not exceed $5 \ldots 7 \%$.

Construction of a mathematical model of the heat processes of laser treatment of the sublayer during at spraying of a triggered coating on the body parts of a gas turbine engine using DOE is constructed. To determine temperature fields occurring in the process of laser treatment of material, software of computational gas dynamics CFX version 15.0 and supercomputer "Sergey Korolev" was used. Temperature dependence of heat conductivity for the coating of the Ni-Al alloy produced by plasma spraying was determined. Alloy heat capacity was calculated based on additive rule. Temperature dependence of the absorption coefficient at CO2-laser treatment was determined.

\section{Acknowledgements}

This work was supported by the Ministry of Education and Science of the Russian Federation as part of the Program "Research and development on priority directions of scientific-technological complex of Russia for 2014-2020" within the project RFMEFI57815X0131

\section{References}

[1] Tucker RC Jr. Thermal spray coatings: Broad and growing applications. Int. J. Powder Metall 2002; 38(7): 45-53.

[2] Batra U. Thermal spray coating of abradable Ni based composite . Surf. Eng. 2009; 25(4): 284-286.

[3] Jin Y, Qian Z, Wang C, Yue J, Li K. Process optimization of plasma spraying Ni-based alloy coating. Heat Treatment of Metals 2013 ; 38(4): 104-108.

[4] Molins R, Normand B, Rannou G, Hannoyer B, Liao H. Interlamellar boundary characterization in Ni-based alloy thermally sprayed coating. Mat. Sci. Eng. A-Struct. 2003; 351(1-2): 325-333.

[5] Wang J-H, Friesel M, Willander M, Warren R. Microstructure of Ni-based self-fluxing alloy sprayed coating. J. Iron Steel Res. Int. 2005; 12(2): 56-59. 
Computer Optics and Nanophotonics / S.P. Murzin, A.Yu. Tisarev, M.V. Blokhin, S.A. Afanasiev

[6] Zhang XC, Xu BS, Xuan FZ, Tu ST, Wang HD, Wu YX. Porosity and effective mechanical properties of plasma-sprayed Ni-based alloy coatings. Appl. Surf. Sci. 2009; 255(8): 4362-4371.

[7] Skulev H, Malinov S, Basheer PAM, Sha W. Modifications of phases, microstructure and hardness of Ni-based alloy plasma coatings due to thermal treatment. Surface and Coatings Technology 2004; 185(1): 18-29.

[8] Kromer R, Costil S, Cormier J, Courapied D, Berthe L, Peyre P, Boustie M. Laser surface patterning to enhance adhesion of plasma sprayed coatings. Surface and Coatings Technology 2015; 278: 171-182.

[9] Liu F, Liu C-S, Tao X-Q, Chen S-Y. Ni-based alloy cladding on copper crystallizer surface by laser. Dongbei Daxue Xuebao. Journal of Northeastern University 2006; 27(10): 1106-1109.

[10] Felgueroso D, Vijande R, Cuetos JM, Tucho R, Hernández A. Parallel laser melted tracks: Effects on the wear behaviour of plasma-sprayed Ni-based coatings. Wear 2008; 264(4): 257-263.

[11] Kromer R, Costil S, Cormier J, Courapied D, Berthe L, Peyre P, Boustie M. Laser surface patterning to enhance adhesion of plasma sprayed coatings. Surface and Coatings Technology 2015; 278: 171-182.

[12] Murzin SP. Formation of structures in materials by laser treatment to enhance the performance characteristics of aircraft engine parts. Computer Optics 2016; 40(3): 353-359. DOI: 10.18287/2412-6179-2016-40-3- 353-359.

[13] Doskolovich LL, Khonina SN, Kotlyar VV, Nikolsky IV, Soifer VA, Uspleniev GV. Focusators into a ring. Opt. Quant. Electron. 1993; $25(11): 801-814$.

[14] Khonina SN, Kotlyar VV, Skidanov RV, Soifer VA. Levelling the focal spot intensity of the focused Gaussian beam. J. Mod. Optic 2000; 47(5): 883-904.

[15] Doskolovich LL, Kazansky NL, Kharitonov SI, Soifer VA. A method of designing diffractive optical elements focusing into plane areas. J. Mod. Optic 1996; 43(7): 1423-1433.

[16] Soifer V. Computer Design of Diffractive Optics. UK, USA, India, Russia: Ed., Cambridge International Science Publishing Ltd. \& Woodhead Pub. Ltd., $2012 ; 896 \mathrm{p}$.

[17] Kharitonov SI, Doskolovich LL, Kazanskiy NL. Solving the inverse problem of focusing laser radiation in a plane region using geometrical optics. Computer Optics 2016; 40(4): 439-450. DOI: 10.18287/2412-6179-2016-40-4-439-450.

[18] Murzin SP. The research of intensification's expedients for nanoporous structures formation in metal materials by the selective laser sublimation of alloy's components. Computer Optics 2011; 35(2): 175-179.

[19] Murzin SP. Local laser annealing for aluminium alloy parts. Laser. Eng. 2016; 33(1-3): 67-76.

[20] Murzin SP, Balyakin VB. Microstructuring the surface of silicon carbide ceramic by laser action for reducing friction losses in rolling bearings. Opt. Laser Technol. 2017; 88: 96-98.

[21] Murzin SP. Formation of nanoporous structures in metallic materials by pulse-periodic laser treatment. Opt. Laser Technol. 2015; 72: 48-52. 